\title{
Combination of Human Leukocyte Antigen and Killer Cell Immunoglobulin-Like Receptor Genetic Background Influences the Onset Age of Hepatocellular Carcinoma in Male Patients with Hepatitis B Virus Infection
}

\author{
Ning Pan, ${ }^{1,2}$ Jie Qiu, ${ }^{2,3}$ Hang Sun,, Fengqin Miao, ${ }^{1,2}$ Qian Shi, ${ }^{2}$ Jinhuan Xu, ${ }^{2}$ Wei Jiang, \\ Hui Jin, ${ }^{4}$ Wei Xie, ${ }^{1,2}$ Youji He, ${ }^{1}$ and Jianqiong Zhang ${ }^{1,2}$ \\ ${ }^{1}$ Department of Pathogenic Biology and Immunology, Southeast University Medical School, 87 Dingjiaqiao Road, \\ Nanjing, Jiangsu 210009, China \\ ${ }^{2}$ Key Laboratory of Developmental Genes and Human Disease, Ministry of Education, Southeast University, Nanjing, \\ Jiangsu 210009, China \\ ${ }^{3}$ The Second Affiliated Hospital of Southeast University, Nanjing, Jiangsu 210000, China \\ ${ }^{4}$ Department of Epidemiology, School of Public Health, Southeast University, Nanjing, Jiangsu 210009, China
}

Correspondence should be addressed to Youji He; heyouji@yahoo.com and Jianqiong Zhang; zhjq@seu.edu.cn

Received 15 July 2013; Revised 17 September 2013; Accepted 17 September 2013

Academic Editor: Saied Mirshahidi

Copyright (C) 2013 Ning Pan et al. This is an open access article distributed under the Creative Commons Attribution License, which permits unrestricted use, distribution, and reproduction in any medium, provided the original work is properly cited.

\begin{abstract}
To investigate whether killer cell immunoglobulin-like receptor (KIR) and human leukocyte antigen (HLA) genetic background could influence the onset age of hepatocellular carcinoma (HCC) in patients with hepatitis B virus (HBV) infection, one hundred and seventy-one males with HBV-related HCC were enrolled. The presence of 12 loci of KIR was detected individually. HLA-A, -B, and $-C$ loci were genotyped with high resolution by a routine sequence-based typing method. The effect of each KIR locus, $H L A$ ligand, and HLA-KIR combination was examined individually by Kaplan-Meier (KM) analysis. Multivariate Cox hazard regression model was also applied. We identified C1C1-KIR2DS2/2DL2 as an independent risk factor for earlier onset age of HCC (median onset age was 44 for C1C1-KIR2DS2/2DL2 positive patients compared to 50 for negative patients, $P=0.04$ for KM analysis; HR = $1.70, P=0.004$ for multivariate Cox model). We conclude that KIR and HLA genetic background can influence the onset age of HCC in male patients with HBV infection. This study may be useful to improve the current HCC surveillance program in HBVinfected patients. Our findings also suggest an important role of natural killer cells (or other KIR-expressing cells) in the progress of HBV-related HCC development.
\end{abstract}

\section{Introduction}

Primary liver cancer, particularly hepatocellular carcinoma (HCC), is the fifth most common cancer worldwide and the third most common cause of cancer mortality. The curative therapy for HCC is surgical resection, for which only the patients with early stage tumor are eligible. However, most cases of HCC patients are detected in their late stages and become ineligible for surgical resection. Globally, more than $50 \%$ of HCC are due to persistent hepatitis B virus (HBV) infection. In the hyperendemic areas of HCC, such as China and Africa, chronic HBV infection contributes to at least
$80 \%$ of HCC cases [1]. Periodic screening for HCC in HBVinfected patients has been practiced widely and been found to be useful in detecting HCC at early stage $[2,3]$. However, the current HCC screening is reported as borderline cost effective in the Asia-Pacific region, and the optimal screening program remains to be established $[4,5]$. One of the controversial issues is that when to begin this periodic screening in HBV infectors due to a wide variety of onset age of HCC. Knowledge on the factors that influence the onset age of HCC will improve current HCC surveillance program in HBVinfected patients. 
Accumulating evidence from the last decade suggests that natural killer (NK) cells play an important role not only in controlling viral hepatitis but also in contributing to the pathogenesis of liver injury and inflammation [6]. For example, NK cell activation has been reported to play a critical role in liver inflammation during chronic HBV infection both in HBV transgenic mice and in HBV-infected patients [79]. Since persistent inflammation has been recognized as a driving force in HCC genesis [10, 11], NK cell activation may also be involved in HBV-related HCC development.

Killer cell immunoglobulin-like receptors (KIR) are involved in the regulation of NK cell activation through recognition of their human leukocyte antigen (HLA) class I ligands. This family of receptors consists of both activating and inhibitory allotypes. KIRs function can be predicted from the length of the cytoplasmic domain, where long receptors $(K I R 2 D L / K I R 3 D L)$ are generally inhibitory and all short receptors (KIR2DS/KIR3DS) are activating. For inhibitory $K I R s$, the major ligands are $H L A-C$ molecules. In terms of $K I R$ recognition, all the allelic variants of $H L A-C$ can be divided into two groups on the basis of alternative amino acids at position 80 of the extracellular domain. HLA-C group 1 alleles $(H L A-C 1)$ have an asparagine at position 80 , whereas $H L A-C$ group 2 alleles $(H L A-C 2)$ have a lysine at this position. KIR2DL1 recognizes $H L A-C 2$ molecules, whereas KIR2DL2 and KIR2DL3 prefer HLA-C1 molecules [12]. Some $H L A-B$ and $H L A-A$ alleles containing a homologous motif termed $B w 4$ are known to bind to KIR3DL1 $[13,14]$. Besides, some $H L A$ class I molecules are reported as putative ligands (HLA-C1-KIR2DS2, HLA-C2-KIR2DS1, HLA-Bw4-KIR3DS1, and HLA-A3/A11-KIR3DL2) [15-18].

It has been clearly demonstrated that the strength of HLA-KIR interactions has functional significance and can influence disease susceptibility [19]. We found that the polymorphisms of KIR and HLA class I loci were associated with $\mathrm{HCC}$ occurrence in $\mathrm{HBV}$-infected patients in a casecontrol study $[20,21]$. We wondered whether the KIR and $H L A$ genetic background could also influence the onset age of HBV-related HCC. HCC is a disease with a strong male dominance, and male patients usually have earlier onset age compared to female patients [1]. In this study, we focused on males and investigated the influence of KIR and HLA genetic background on the onset age of HBV-related HCC.

\section{Methods}

2.1. Patients. One hundred and seventy-one unrelated patients were enrolled from the Second Affiliated Hospital of Southeast University. Among them, 114 patients were enrolled from 2005 to 2007, and 57 patients were enrolled from 2011 to 2012. The patients were selected according to the following criteria: (a) being diagnosed as having primary HCC; (b) being males; (c) hepatitis B surface antigen (HBsAg) being positive for more than six months; (d) having hepatic ultrasonography within the past 12 months before they were diagnosed as having HCC; and (e) being members of Han population and living in the same geographical area. The exclusion criteria include: (a) being positive for other hepatitis viruses serum markers (hepatitis A virus IgM, hepatitis $C$ virus antibody, hepatitis $D$ virus antigen, hepatitis $\mathrm{D}$ virus antibody, and hepatitis E virus $\operatorname{IgM}$ ) and human immunodeficiency virus antibody; (b) having an indication of autoimmune disease. All diagnoses of HCC were defined by clinical and biological criteria and confirmed by imaging technologies (defined as 1 or more tumoral nodules by computed tomography and ultrasonography). Eighty-six patients were also included in our previous case-control study [19]. The protocol was approved by the ethics committee of the Second Affiliated Hospital of Southeast University, and all the patients provided written, informed consent before enrollment.

2.2. Extraction of Genomic DNA. Genomic DNA was extracted from peripheral blood mononuclear cells by a standard salting-out method.

2.3. KIR Genotyping. PCR amplification with the primers specific for each locus of the following inhibitory KIR genes: 2DL1, 2DL2, 2DL3, 2DL5, and 3DL1 and activating KIR genes: 2DS1, 2DS2, 2DS3, 2DS4(f) (the full-length form of 2DS4), $2 D S 4$ (d) (the 22 bp-deletion mutant form of 2DS4), 2DS5, and $3 D S 1$ was performed as described in previous reports [20, 22]. The internal positive control primers for the fragment of the framework gene KIR2DL4 were included in each PCR reaction. All primer sequences and amplification conditions are available upon request.

2.4. HLA Genotyping. HLA-A, $-B$, and $-C$ were genotyped with high resolution by a routine sequence-based typing method [23]. Exons 2 and 3 of $H L A-A, H L A-B$, and $H L A-$ $C$ loci were amplified from genomic DNA by PCR using the locus-specific primers as described [23]. Sequencing reactions were performed using the BigDye Terminator v3.1 Cycle Sequencing Ready Reaction Kit (Applied Biosystems). Exons 2 and 3 of each locus were sequenced in both forward and reverse directions using a 3730XL DNA Analyzer (Applied Biosystems, Foster City, CA). The sequences were then analyzed using online dbMHC SBT typing tool [24]. One novel HLA class I allele was identified in this population. Nucleotide sequence of new allele has been submitted to the GenBank nucleotide sequence database and is available under the accession number EF468681 [25].

2.5. Statistical Methods. The effects of each KIR locus, HLA ligand, and $H L A-K I R$ combination were examined individually by Kaplan-Meier (KM) analysis. $P$ values were determined using Log Rank test for KM analysis. Univariate Cox proportional hazard regression model was applied to each demographic and clinical variable individually (see Table S3 in Supplementary Material available online at http://dx.doi.org/10.1155/2013/874514). After that, multivariate Cox regression analysis was employed to the genetic factors with $P$ value less than 0.05 and the clinical variables with $P$ value less than 0.5 (Table 4 ). $P$ values generated through Cox modeling were supported with hazard ratios (HR) and 95\% confidence intervals (CI). HLA-C genotype 
frequencies were checked for Hardy-Weinberg equilibrium using Pearson's $\chi^{2}$ test. All the median ages were estimated by $\mathrm{KM}$ analysis. The final Cox regression model (Table 4 ) included 161 cases because the genotyping of some loci could not be accomplished for some cases due to lack of DNA. All statistical analyses were performed using SPSS software (Version 11.0 Chicago IL, SPSS Inc.).

\section{Results}

3.1. Patients' Demographic and Clinical Characteristics (Table 1). All HCC patients selected were males with history of chronic hepatitis B, free of other hepatic virus coinfection, and with no indication of autoimmune disease. One hundred and forty-two patients $(83.0 \%)$ were diagnosed as having cirrhosis, which was consistent with previous reports [26]. Sixty-three patients $(36.8 \%)$ were at TNM stage III or IV when they were diagnosed as HCC. The HBeAg status and HBV DNA level presented in Table 1 were tested when the patients were diagnosed as having HCC. However, only 70 patients were quantified for HBV DNA in sera when they were diagnosed as having HCC. The onset age value was determined from KM survival-time data, with the 25th, 50th, and 75th percentiles of cancer free survival time being reported. The median onset age, defined as the age at which $50 \%$ of the population is cancer free, was 50 years. The distribution of onset ages of HCC is comparable with the recent report in Shanghai population [27].

3.2. Association of the Onset Age with KIR Polymorphisms (Table 2). Twelve KIR genes were detected in this population. The result showed that KIR2DL1 (97.7\%), KIR2DL3 (97.7\%), and KIR3DL1 (98.2\%) were present in nearly all individuals. The frequencies of other KIR loci varied from $15.2 \%$ to $83.6 \%$. $\mathrm{KM}$ analyses were performed on each of the KIR genes individually. No statistical significant result was found on any given KIR locus (Table 2).

3.3. Association of the Onset Age with HLA Ligands and HLAKIR Combinations. One hundred and forty-nine HLA class I alleles (37 HLA-A alleles, $60 H L A-B$ alleles, and $52 H L A-C$ alleles) were identified in these patients. To explore whether $H L A$ ligands for KIR could influence the onset age of HBVrelated HCC, we grouped HLA alleles according to KIR ligand as $B w 4, B w 4 T, B w 4 I, H L A-C 1$, and $H L A-C 2$. KM analyses were performed on Bw4,Bw4T, Bw4I, HLA-C1C1, HLA$\mathrm{C1C2}$, and $\mathrm{HLA}-\mathrm{C} 2 \mathrm{C} 2$ individually. The frequencies of $\mathrm{HLA}-\mathrm{C}$ genotypes were consistent with Hardy-Weinberg equilibrium $(P=0.44)$. Putative ligands of $H L A-A 3$ and $-A 11$ were also analyzed. However, no significant result was found (Table S1). Then, we tested the effects of Bw4-KIR ligand-receptor (or putative ligand-receptor) combinations, including $B w 4$ KIR3DL1, Bw4T-KIR3DL1, Bw4I-KIR3DL1, Bw4-KIR3DS1, $B w 4 T-K I R 3 D S 1$, and Bw4I-KIR3DS1, on the onset age of HCC by KM analysis individually. No significant result was found (Table S2).

The effect of each HLA-C-KIR ligand-receptor (or putative ligand-receptor) combination on the onset age of HCC
TABLE 1: Demographic and clinical characteristics of 171 male HCC patients.

\begin{tabular}{|c|c|c|}
\hline & Number of cases & Percentage \\
\hline \multicolumn{3}{|l|}{ Age of onset ${ }^{\dagger}$} \\
\hline Median $(\mathrm{IQR})^{\ddagger}$ & $50(42 \sim 57)$ & - \\
\hline Range & $24 \sim 74$ & - \\
\hline Cirrhosis & 142 & $83.0 . \%$ \\
\hline TNM stage III or IV & 63 & $36.8 \%$ \\
\hline HBsAg positive & 171 & $100 \%$ \\
\hline HBeAg positive & 60 & $35.1 \%$ \\
\hline \multicolumn{3}{|l|}{ HBV DNA copy number ${ }^{\dagger \diamond}$} \\
\hline Mean & $2.3 E+06$ & - \\
\hline Range & $0 \sim 4.8 E+07$ & - \\
\hline Family history of HCC & 9 & $5.3 \%$ \\
\hline $\begin{array}{l}\text { Family history of HBV-related } \\
\text { diseases }\end{array}$ & 43 & $25.1 \%$ \\
\hline $\begin{array}{l}\text { Alcohol consumption ( } \geq 1 \text { drink } \\
\text { per week) }\end{array}$ & 20 & $11.7 \%$ \\
\hline
\end{tabular}

${ }^{\dagger}$ Age of first diagnosis for HCC.

${ }^{\ddagger}$ Age is presented as median, interquartile range (IQR), which are determined by KM estimates.

${ }^{\S} 70$ patients were quantified for HBV DNA when they were diagnosed as HCC.

'HBV-related diseases include HBV-related hepatitis, cirrhosis, liver failure, and HCC.

was then analyzed. There was high linkage disequilibrium in KIR2DS2 and KIR2DL2 in our patients as in all populations tested to date (http://www.allelefrequencies.net/). Because our data did not allow to distinguish the effects between $\mathrm{ClCl}$ KIR2DS2 and C1C1-KIR2DL2, we combined C1C1-KIR2DS2 and C1C1-KIR2DL2 as HLA-C1C1 + KIR2DS2 or/and KIR2DL2 (C1C1-KIR2DS2/2DL2). C1C1-KIR2DS2/2DL2 was found to be associated with earlier onset age of HCC (the median age for the C1C1-KIR2DS2/2DL2 positive patients was 44 years, compared to 50 years for the C1C1KIR2DS2/2DL2 negative patients, $P=0.004)$. No statistical significance was found on the other HLA-C-KIR ligandreceptor combinations (Table 3).

The effects of each demographic and clinical variable (including cirrhosis status, HBeAg status, TNM stage, family history of HBV-related diseases, and alcohol consumption) on the onset age of HCC were tested by univariate Cox model analysis (Table S3). To maintain statistical power, the demographic and clinical variables with $P$ value less than 0.5 were included as the covariables in the multivariate Cox hazard regression model to analyze the effect of $\mathrm{ClCl}$ KIR2DS2/2DL2. The result showed that C1C1-KIR2DS2/2DL2 was an independent risk factor for earlier onset age of HCC $(\mathrm{HR}=1.70,95 \% \mathrm{CI}=1.01-2.85, P=0.04$, Table 4$)$.

\section{Discussion}

HCC surveillance in patients with chronic HBV infection has been recommended by various regional liver societies. Hepatic ultrasonography and alpha fetoprotein test every 6 months are the preferred program in China [28]. Although 
TABLE 2: Effects of KIR genes and genotypes on the onset age of HCC by $\mathrm{KM}$ analysis.

\begin{tabular}{|c|c|c|c|c|}
\hline $\begin{array}{l}\text { KIR gene and } \\
\text { genotype }\end{array}$ & & $n(\%)$ & Median age & $P$ value \\
\hline \multirow{2}{*}{$2 D L 1$} & Negative & $4(2.3)$ & 45 & \multirow{2}{*}{0.82} \\
\hline & Positive & 167 (97.7) & 50 & \\
\hline \multirow{2}{*}{$2 D L 2$} & Negative & $137(80.1)$ & 50 & \multirow{2}{*}{0.09} \\
\hline & Positive & $34(19.9))$ & 49 & \\
\hline \multirow{2}{*}{$2 D L 3$} & Negative & $4(2.3)$ & 45 & \multirow{2}{*}{0.81} \\
\hline & Positive & $167(97.7))$ & 50 & \\
\hline \multirow{2}{*}{$2 D L 5$} & Negative & $103(60.2)$ & 50 & \multirow{2}{*}{0.67} \\
\hline & Positive & $68(39.8)$ & 50 & \\
\hline \multirow{2}{*}{$3 D L 1$} & Negative & $3(1.8)$ & 41 & \multirow{2}{*}{0.68} \\
\hline & Positive & $168(98.2)$ & 50 & \\
\hline \multirow{2}{*}{$2 D S 1$} & Negative & $109(63.7)$ & 50 & \multirow{2}{*}{0.49} \\
\hline & Positive & $62(36.3)$ & 49 & \\
\hline \multirow{2}{*}{$2 D S 2$} & Negative & $139(81.3)$ & 50 & \multirow{2}{*}{0.09} \\
\hline & Positive & $32(18.7)$ & 50 & \\
\hline \multirow{2}{*}{$2 D S 3$} & Negative & $145(84.8)$ & 50 & \multirow{2}{*}{0.07} \\
\hline & Positive & $26(15.2)$ & 49 & \\
\hline \multirow{2}{*}{$2 D S 4(f)$} & Negative & $28(16.4)$ & 49 & \multirow{2}{*}{0.83} \\
\hline & Positive & $143(83.6)$ & 50 & \\
\hline \multirow{2}{*}{$2 D S 4(d)$} & Negative & $116(67.8)$ & 50 & \multirow{2}{*}{0.97} \\
\hline & Positive & $55(32.2)$ & 49 & \\
\hline \multirow{2}{*}{$2 D S 5$} & Negative & $124(72.5)$ & 50 & \multirow{2}{*}{0.50} \\
\hline & Positive & 47 (27.5) & 51 & \\
\hline \multirow{2}{*}{$3 D S 1$} & Negative & $108(63.2)$ & 49 & \multirow{2}{*}{0.98} \\
\hline & Positive & $63(36.8)$ & 50 & \\
\hline \multirow{2}{*}{ KIR genotype } & AA & $84(49.1)$ & 50 & \multirow{2}{*}{0.42} \\
\hline & $\mathrm{BX}$ & 87 (50.9) & 50 & \\
\hline
\end{tabular}

this periodic screening has been practiced widely, its benefits remain uncertain. Therefore, an optimal HCC surveillance program with acceptable cost effectiveness is needed, particularly for at-risk populations $[4,29]$. One of the controversial issues is that when to begin this periodic screening in HBVinfected patients. The American Association for Study of Liver Disease recommends that, for noncirrhotic patients with hepatitis $B$, males above the age of 40 years and females above the age of 50 years are appropriate candidates for HCC surveillance [30]. The Asia-Pacific Association for Study of Liver Disease recommends the HCC surveillance program for high-risk patients with chronic hepatitis B (especially those who aged $>30$ years with serum HBV DNA levels $>20$ $000 \mathrm{IU} / \mathrm{mL}$ ) in the absence of a known diagnosis of cirrhosis [5]. The Ministry of Health of the People's Republic of China recommends lately that, for $\mathrm{HBV}$-infected patients, males above the age of 40 years and females above the age of 50 years are appropriate candidates for HCC surveillance [28]. Knowledge on the factors that influence the age of HCC onset will provide basis for the improvement of the current HCC surveillance programs. HCC occurs mainly in men. Male : female ratios between 3:1 and 4:1 are reported in East
TABLE 3: Effects of HLA-C-KIR ligand-receptor combinations on the onset age of HCC by KM analysis.

\begin{tabular}{lcccc}
\hline HLA-KIR combination & & $n$ & Median age & $P$ value \\
\hline C1C1-KIR2DL3 & Negative & 40 & 50 & 0.81 \\
& Positive & 98 & 47 & \\
C1C2-KIR2DL3 & Negative & 109 & 48 & 0.78 \\
& Positive & 29 & 50 & \\
C1C2-KIR2DL1 & Negative & 110 & 49 & 0.65 \\
& Positive & 28 & 50 & \\
C2C2-KIR2DL1 & Negative & 131 & 49 & 0.81 \\
& Positive & 7 & 50 & \\
C1C1-KIR2DS2/2DL2 & Negative & 141 & 50 & $0.004^{* *}$ \\
& Positive & 20 & 44 & \\
C1C2-KIR2DS2/2DL2 & Negative & 155 & 49 & 0.53 \\
& Positive & 8 & 53 & \\
C1C2-KIR2DS1 & Negative & 134 & 50 & 0.68 \\
C2C2-KIR2DS1 & Positive & 7 & 50 & \\
& Negative & 138 & 50 & 0.12 \\
$* *$ P $<0.01$. & Positive & 3 & 43 & \\
& & & &
\end{tabular}

TABle 4: Multivariate Cox model survival analysis of C1C1+2DS2/2DL2 and other factors on the onset age of HCC.

\begin{tabular}{lccc}
\hline Factor & $P$ value & HR & $95 \%$ CI \\
\hline C1C1+2DS2/2DL2 & $0.04^{*}$ & 1.70 & $1.01-2.85$ \\
Cirrhosis & 0.03 & 0.59 & $0.36-0.96$ \\
HBeAg positive & 0.39 & 1.19 & $0.80-1.77$ \\
Family history of HBV-related diseases & 0.23 & 1.30 & $0.85-2.01$ \\
\hline
\end{tabular}

${ }^{*} P<0.05$.

China $[1,20,27]$. In this study, we examined the KIR and HLA genetic background in 171 male patients. We found that the median onset age of HCC was 6 years earlier in patients with a particular HLA-KIR combination of C1C1-KIR2DS2/2DL2 than that of the patients without this combination (Table 4). The patients with C1C1-KIR2DS2/2DL2 accounted for about $12 \%$ of this study cohort. Because both KIR genes and HLA$\mathrm{Cl}$ can be identified by real-time polymerase chain reaction [31], which is economical and time saving, the detection of KIR2DS2/2DL2 and HLA-C1 is easy to be applied in clinical practice. Therefore, this study could help focus earlydetection programs to a two-tiered model for greatest costbenefit ratio.

In our previous case-control study, several $K I R$ and $H L A$ variants, including HLA-C1C1, HLA-Bw4-80I, and $\operatorname{KIR} 2 D S 4(f) /(d)$, were identified as the risk factors for HCC development in the patients with HBV infection. Because all of these risk factors we found had been reported to result in high NK cell functional potential, the data strongly suggested that overactivation of NK cell contributed to HBV-related HCC development [20]. The results of current study support the importance of NK cells (or other KIR expressing cells) in the progress of HBV-related HCC development. We could not distinguish the effect between C1C1-2DS2 and C1C1-2DL2 
because KIR2DS2 and KIR2DL2 were in high linkage disequilibrium. Since KIR2DS2 delivers activating signal while KIR2DL2 delivers inhibiting signal, the correlation itself does not indicate the underlying mechanism. However, since there is little biologic difference between KIR2DL2 and KIR2DL3 (KIR2DL2 and KIR2DL3 are alleles of the same locus with the same HLA ligand) and C1C1-2DL3 has no effect on onset age of HCC ( $P=0.81$, Table 3$)$, it seems more plausible that the activating KIR2DS2 is the biologically relevant predictor.

According to the full combinations of HLA-C genotype and KIR2DS2/2DL2, the population can be divided into four groups: (a) C1C1-2DS2/2DL2, (b) C1C1-2DS2/2DL2-, (c) C2-2DS2/2DL2, and (d) C2-2DS2/2DL2-. Besides C1C1$2 D S 2 / 2 D L 2$, the effects of other three groups on the onset age were also analyzed. The median onset ages for groups C1C1-2DS2/2DL2-, C2-2DS2/2DL2, and C2-2DS2/2DL2- were 49 years, 53 years, and 49 years, respectively. None of the combinations influenced the onset age of HCC significantly ( $P=0.75,0.28$, and 0.31 by $\mathrm{KM}$ analyses, resp.). The median onset age of $C 2-2 D S 2 / 2 D L 2$ group was 9 years older than that of C1C1-2DS2/2DL2 group (53 years versus 44 years, $P=0.03$ by KM analysis). This result supports further that KIR and HLA combinations can influence the onset age of HCC.

To further refine programs for those at highest risk of unresectable HCC, we explored the association between KIR locus, HLA ligand, and HLA-KIR combination with the TNM stages of HCC at diagnosis. However, no statistical significance was found (data not shown).

The determination of HCC onset is rather difficult because most HCC patients are asymptomatic at the early stage of HCC. In general, many HBV-infected patients take regular abdomen ultrasonography in a biannual HCC surveillance program or in an annual routine medical examination. To determine the precise onset of HCC, we selected patients who had taken abdomen ultrasonography within the past 12 months before they were diagnosed as HCC, which means that these patients had not developed HCC (or ultrasonography-detectable HCC) one year before their diagnosis. Therefore, the onset of HCC was defined as the time of first diagnosis in this study.

Virological factors, such as HBeAg status, HBV viral load, $\mathrm{HBV}$ genotype, and $\mathrm{HBV}$ mutations, are reported to influence the HCC risk, although few reports concern the onset age of HCC [32]. Population-based cohort studies support that positive for HBeAg associated with increased HCC risk [33, 34]. Our results showed that positive for $\mathrm{HBeAg}$ at diagnosis had no influence on the onset age of HCC ( $\mathrm{HR}=1.17$, $P=0.38$, Table S3). Level of HBV viraemia from the age of 30 years was reported as an independent risk factor for HCC during the next decade [33]. However, the result of univariate Cox analysis showed that the HBV viral load, at their diagnosis as HCC, had no influence on the onset age of HCC in our study population ( $\mathrm{HR}=1.00, P=0.89$, Table S3). HBV genotype $\mathrm{C}$ in Asian cohorts [35], genotype $\mathrm{F}$ in other populations [36], and some HBV mutations [37] were reported to increase the risk of HCC. One limitation of our study is lack of information of HBV genotype and mutations in the patients.

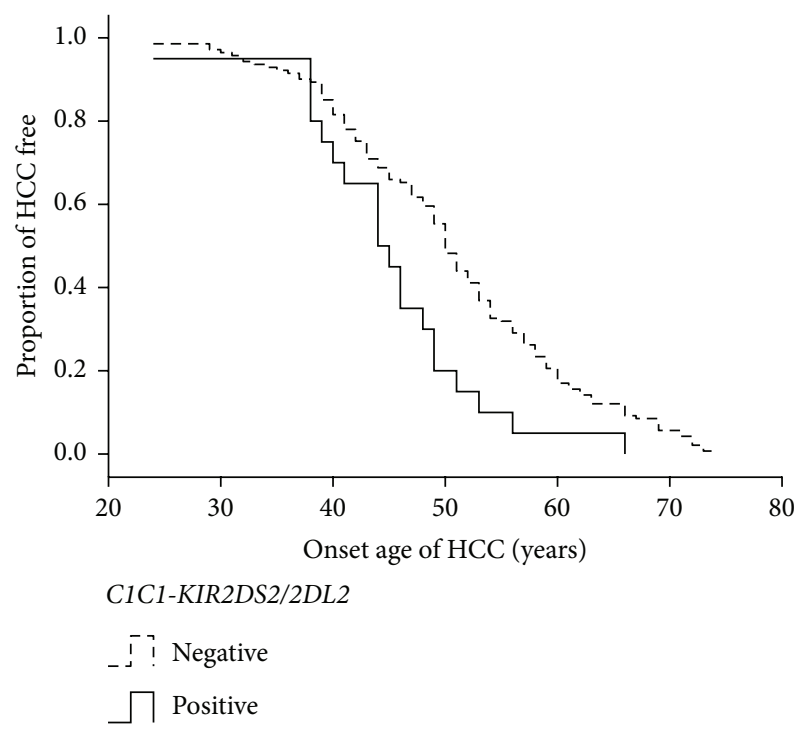

FIGURE 1: The effect of C1C1-KIR2DS2/2DL2 on the onset age of HCC by KM survival analysis. The plot shows the effect of C1C1KIR2DS2/2DL2 on the onset age of HCC in males with HBV infection $(n=161)$. C1C1-KIR2DS2/2DL2 positive patients have earlier onset age of HCC $(P=0.004)$.

\section{Conclusion}

The present study shows that $C 1 C 1-2 D S 2 / 2 D L 2$ is an independent risk factor for earlier onset age of HCC (Figure 1, Table 4) in male patients with HBV infection. To our knowledge, this is the first report on the association between KIR and HLA genetic background and the onset age of HCC. Our findings may be useful to improve the current HCC surveillance program in local HBV-infected patients. Additionally, this observation along with our previous casecontrol study suggests an important role of NK cell (or other $K I R$-expressing cell) in the process of HCC development during HBV infection. Future studies on the mechanism underlying these genetic associations may provide insights for new therapeutic strategies in preventing HCC in HBVinfected patients.

\section{Conflict of Interests}

The authors declare that there is no conflict of interests.

\section{Authors' Contribution}

Ning Pan and Jie Qiu contributed equally to this paper.

\section{Acknowledgments}

This work was supported by the National Natural Science Foundation of China (30571703), the National Natural Science Foundation for Young Scholars of China (81201601), the Science Foundation of Department of Health of Jiangsu Province (H200745), and the Science Foundation of Science and Technology Bureau of Nanjing (200702067). The funders 
had no role in study design, data collection and analysis, decision to publish, or preparation of the paper. The authors would like to thank Dr. Zhizhou He for critical reading of the paper.

\section{References}

[1] H. B. El-Serag, "Hepatocellular carcinoma," The New England Journal of Medicine, vol. 365, no. 12, pp. 1118-1127, 2011.

[2] B. J. McMahon, L. Bulkow, A. Harpster et al., "Screening for hepatocellular carcinoma in Alaska natives infected with chronic hepatitis B: a 16-year population-based study," Hepatology, vol. 32, no. 4 I, pp. 842-846, 2000.

[3] B. Zhang, B. Yang, and Z. Tang, "Randomized controlled trial of screening for hepatocellular carcinoma," Journal of Cancer Research and Clinical Oncology, vol. 130, no. 7, pp. 417-422, 2004.

[4] D. Amarapurkar, K. Han, H. L. Chan, and Y. Ueno, "Application of surveillance programs for hepatocellular carcinoma in the Asia-Pacific Region," Journal of Gastroenterology and Hepatology, vol. 24, no. 6, pp. 955-961, 2009.

[5] G. Farrell, "Prevention of hepatocellular carcinoma in the AsiaPacific region: consensus statements," Journal of Gastroenterology and Hepatology, vol. 25, no. 4, pp. 657-663, 2010.

[6] Z. Tian, Y. Chen, and B. Gao, "Natural killer cells in liver disease," Hepatology, vol. 57, no. 4, pp. 1654-1662, 2013.

[7] C. Dunn, M. Brunetto, G. Reynolds et al., "Cytokines induced during chronic hepatitis B virus infection promote a pathway for NK cell-mediated liver damage," Journal of Experimental Medicine, vol. 204, no. 3, pp. 667-680, 2007.

[8] Y. Chen, R. Sun, W. Jiang, H. Wei, and Z. Tian, "Liver-specific HBsAg transgenic mice are over-sensitive to Poly(I:C)-induced liver injury in NK cell- and IFN- $\gamma$-dependent manner," Journal of Hepatology, vol. 47, no. 2, pp. 183-190, 2007.

[9] E. Trachtenberg, M. Vinson, E. Hayes et al., "HLA class I (A, $\mathrm{B}, \mathrm{C})$ and class II (DRB1, DQA1, DQB1, DPB1) alleles and haplotypes in the Han from southern China," Tissue Antigens, vol. 70, no. 6, pp. 455-463, 2007.

[10] A. Mantovani, P. Allavena, A. Sica, and F. Balkwill, "Cancerrelated inflammation," Nature, vol. 454, no. 7203, pp. 436-444, 2008.

[11] C. Berasain, J. Castillo, M. J. Perugorria, M. U. Latasa, J. Prieto, and M. A. Avila, "Inflammation and liver cancer: new molecular links," Annals of the New York Academy of Sciences, vol. 1155, pp. 206-221, 2009.

[12] L. L. Lanier, "NK cell recognition," Annual Review of Immunology, vol. 23, pp. 225-274, 2005.

[13] J. E. Gumperz, V. Litwin, J. H. Phillips, L. L. Lanier, and P. Parham, "The Bw4 public epitope of HLA-B molecules confers reactivity with natural killer cell clones that express NKB1, a putative HLA receptor," Journal of Experimental Medicine, vol. 181, no. 3, pp. 1133-1144, 1995.

[14] M. Stern, L. Ruggeri, M. Capanni, A. Mancusi, and A. Velardi, "Human leukocyte antigens A23, A24, and A32 but not A25 are ligands for KIR3DL1," Blood, vol. 112, no. 3, pp. 708-710, 2008.

[15] J. H. Chewning, C. N. Gudme, K. C. Hsu, A. Selvakumar, and B. Dupont, "KIR2DS1-positive NK cells mediate alloresponse against the C2 HLA-KIR ligand group in vitro," Journal of Immunology, vol. 179, no. 2, pp. 854-868, 2007.
[16] B. Foley, D. De Santis, L. Lathbury, F. Christiansen, and C. Witt, "KIR2DS1-mediated activation overrides NKG2Amediated inhibition in HLA-C C2-negative individuals," International Immunology, vol. 20, no. 4, pp. 555-563, 2008.

[17] P. Hansasuta, T. Dong, H. Thananchai et al., "Recognition of HLA-A3 and HLA-A11 by KIR3DL2 is peptide-specific," European Journal of Immunology, vol. 34, no. 6, pp. 1673-1679, 2004.

[18] A. Thielens, E. Vivier, and F. Romagné, "NK cell MHC class I specific receptors (KIR): from biology to clinical intervention," Current Opinion in Immunology, vol. 24, no. 2, pp. 239-245, 2012.

[19] P. Kusnierczyk, "Killer cell immunoglobulin-like receptor gene associations with autoimmune and allergic diseases, recurrent spontaneous abortion, and neoplasms," Frontiers in Immunology, vol. 4, article 8, 2013.

[20] N. Pan, W. Jiang, H. Sun et al., "KIR and HLA loci are associated with hepatocellular carcinoma development in patients with hepatitis B virus infection: a case-control study," PLOS ONE, vol. 6, no. 10, Article ID e25682, 2011.

[21] N. Pan, K. Chen, J. Qiu et al., "Human leukocyte antigen class I alleles and haplotypes associated with primary hepatocellular carcinoma in persistent HBV-infected patients," Human Immunology, vol. 74, no. 6, pp. 758-763, 2013.

[22] K. C. Hsu, X. Liu, A. Selvakumar, E. Mickelson, R. J. O’Reilly, and B. Dupont, "Killer Ig-like receptor haplotype analysis by gene content: evidence for genomic diversity with a minimum of six basic framework haplotypes, each with multiple subsets," Journal of Immunology, vol. 169, no. 9, pp. 5118-5129, 2002.

[23] P. P. J. Dunn, S. T. Cox, and A. Little, "Sequencing protocols for detection of HLA class I polymorphism," Methods in Molecular Biology, vol. 210, pp. 191-222, 2003.

[24] W. Helmberg, R. Dunivin, and M. Feolo, "The sequencingbased typing tool of dbMHC: typing highly polymorphic gene sequences," Nucleic Acids Research, vol. 32, pp. W173-W175, 2004.

[25] W. Jiang, J. Q. Zhang, N. Pan, J. H. Xu, and W. Xie, "Identification of a novel HLA-A allele, HLA-A $* 9216$," Tissue Antigens, vol. 71, no. 5, pp. 479-480, 2008.

[26] H. B. El-Serag and K. L. Rudolph, "Hepatocellular carcinoma: epidemiology and molecular carcinogenesis," Gastroenterology, vol. 132, no. 7, pp. 2557-2576, 2007.

[27] S. Gao, W. Yang, F. Bray et al., "Declining rates of hepatocellular carcinoma in urban Shanghai: incidence trends in 1976-2005," European Journal of Epidemiology, vol. 27, no. 1, pp. 39-46, 2012.

[28] China MoHotPsRo, "Diagnosis, management, and treatment of hepatocellular carcinoma (v2011)," Journal of Clinical Hepatology, vol. 16, no. 10, pp. 1141-1159, 2011.

[29] E. C. Doo, J. H. Hoofnagle, and G. P. Rodgers, "NIH consensus development conference: management of hepatitis B. Introduction," Hepatology, vol. 49, supplement, no. 5, pp. S1-S3, 2009.

[30] J. Bruix and M. Sherman, "Management of hepatocellular carcinoma," Hepatology, vol. 42, no. 5, pp. 1208-1236, 2005.

[31] H. A. Hong, A. S. Loubser, D. de Assis Rosa et al., "Killercell immunoglobulin-like receptor genotyping and HLA killercell immunoglobulin-like receptor-ligand identification by realtime polymerase chain reaction," Tissue Antigens, vol. 78, no. 3, pp. 185-194, 2011.

[32] B. C. Taylor, J. Yuan, T. A. Shamliyan, A. Shaukat, R. L. Kane, and T. J. Wilt, "Clinical outcomes in adults with chronic hepatitis B in association with patient and viral characteristics: a systematic 
review of evidence," Hepatology, vol. 49, supplement, no. 5, pp. S85-S95, 2009.

[33] C. Chen, H. Yang, J. Su et al., "Risk of hepatocellular carcinoma across a biological gradient of serum hepatitis B virus DNA Level," Journal of the American Medical Association, vol. 295, no. 1, pp. 65-73, 2006.

[34] B. J. McMahon, P. Holck, L. Bulkow, and M. Snowball, "Serologic and clinical outcomes of 1536 Alaska natives chronically infected with hepatitis B virus," Annals of Internal Medicine, vol. 135, no. 9, pp. 759-768, 2001.

[35] H. L.-Y. Chan, A. Y. Hui, M. L. Wong et al., "Genotype C hepatitis $\mathrm{B}$ virus infection is associated with an increased risk of hepatocellular carcinoma," Gut, vol. 53, no. 10, pp. 1494-1498, 2004.

[36] S. E. Livingston, J. P. Simonetti, B. J. McMahon et al., "Hepatitis B virus genotypes in Alaska Native people with hepatocellular carcinoma: preponderance of genotype F," Journal of Infectious Diseases, vol. 195, no. 1, pp. 5-11, 2007.

[37] M. J. Tong, L. M. Blatt, J. Kao, J. T. Cheng, and W. G. Corey, "Basal core promoter T1762/A1764 and precore A1896 gene mutations in hepatitis B surface antigen-positive hepatocellular carcinoma: a comparison with chronic carriers," Liver International, vol. 27, no. 10, pp. 1356-1363, 2007. 


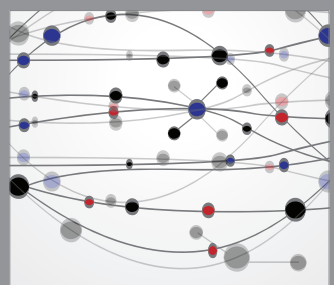

The Scientific World Journal
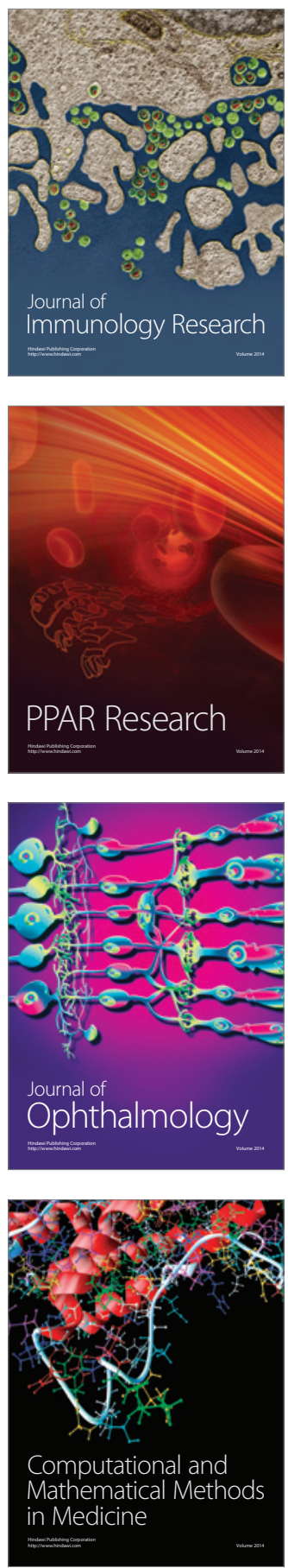

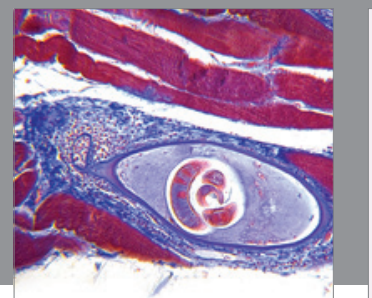

Gastroenterology

Research and Practice
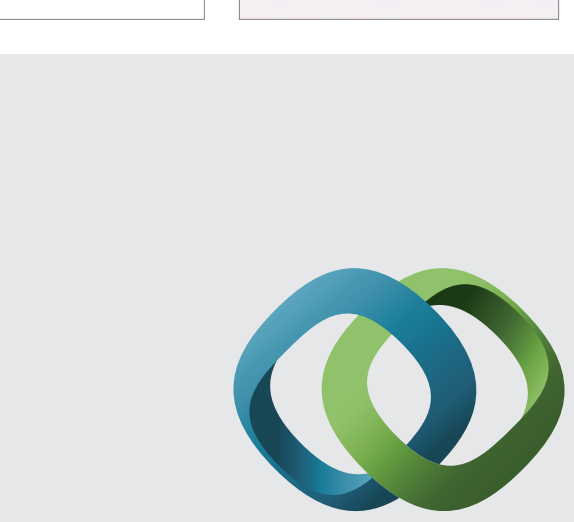

\section{Hindawi}

Submit your manuscripts at

http://www.hindawi.com
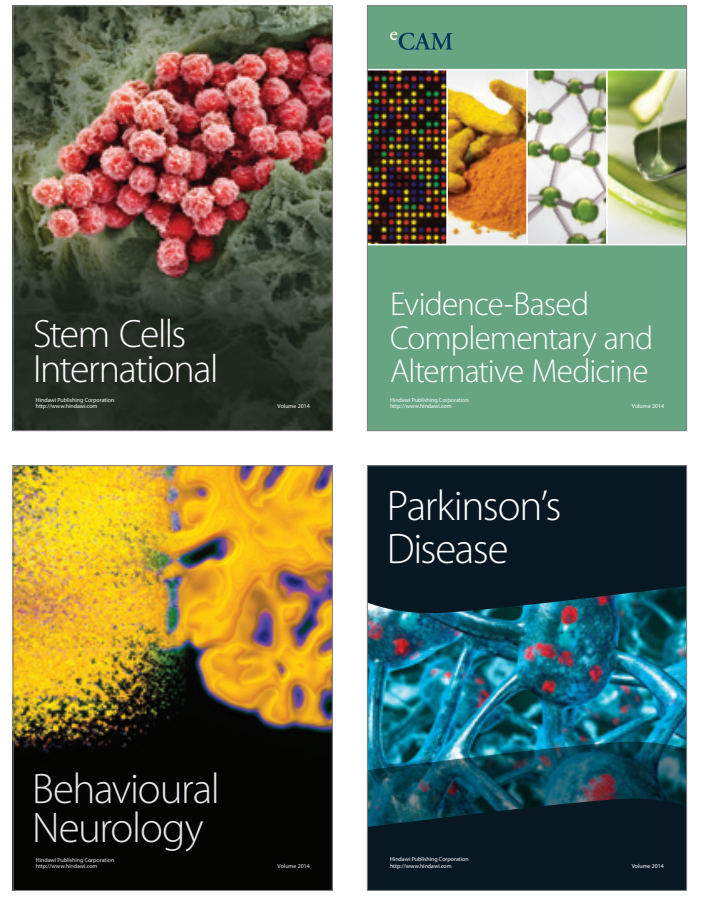
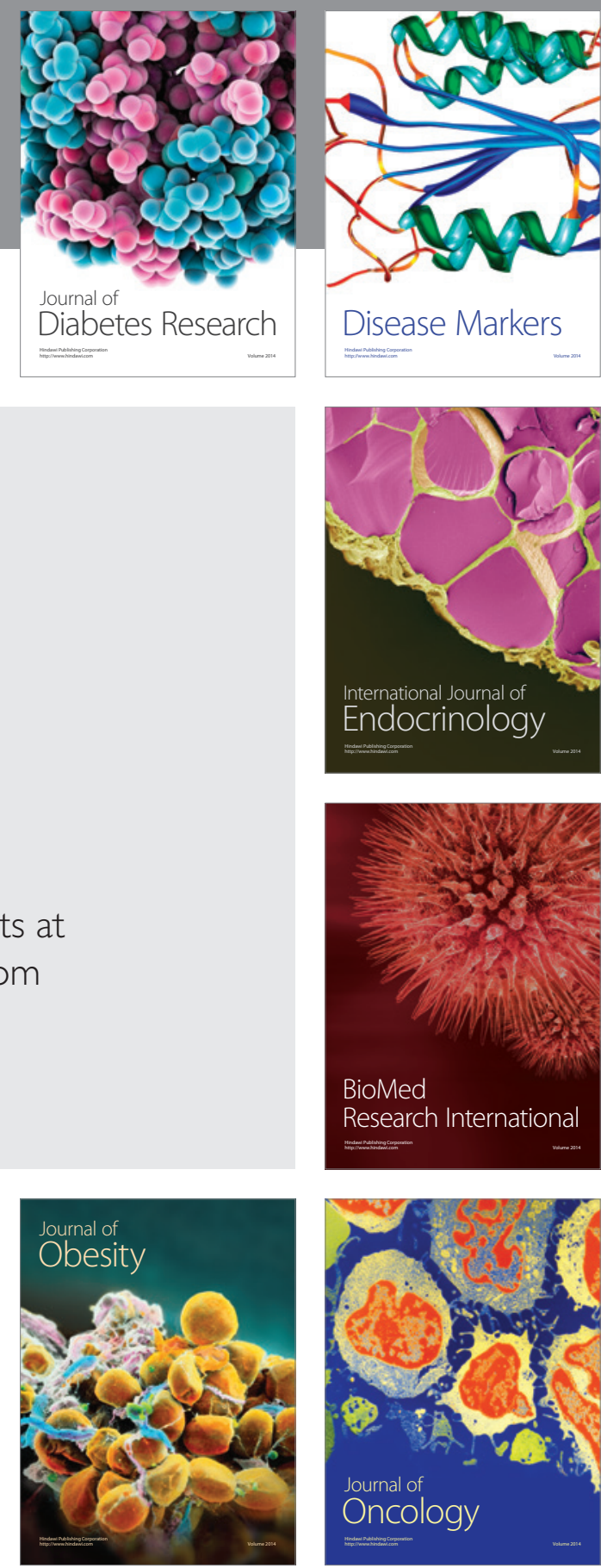

Disease Markers
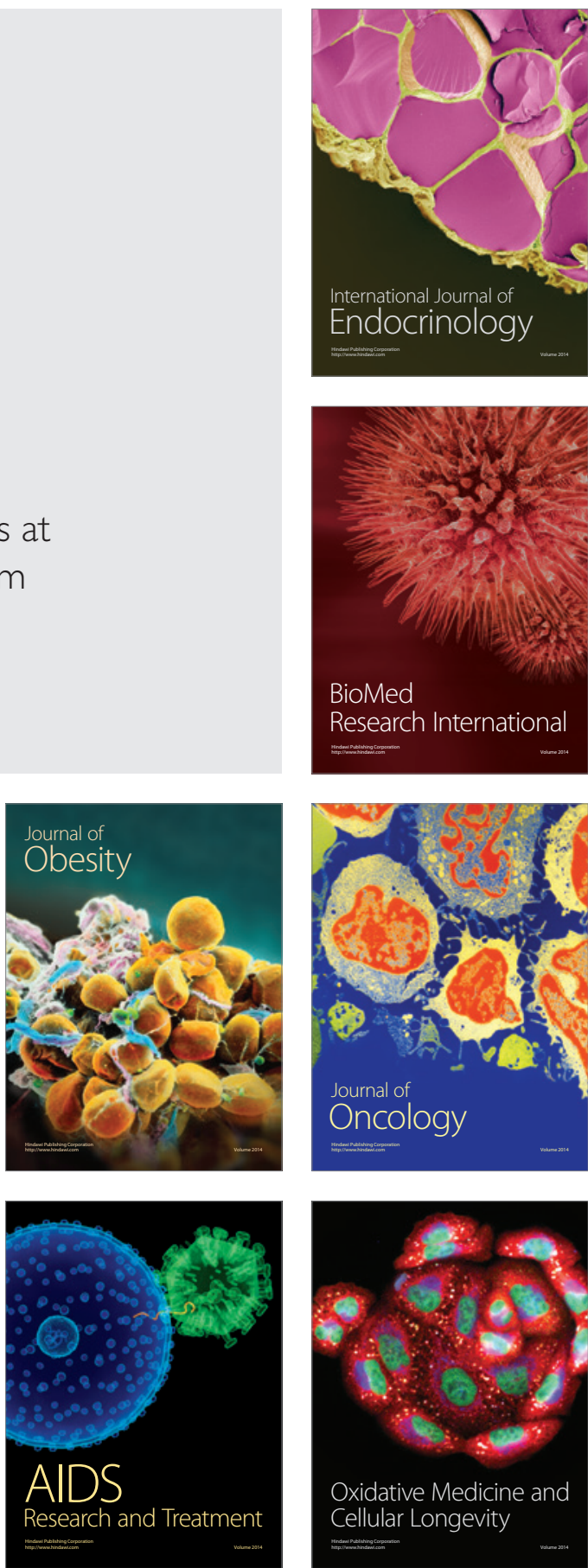